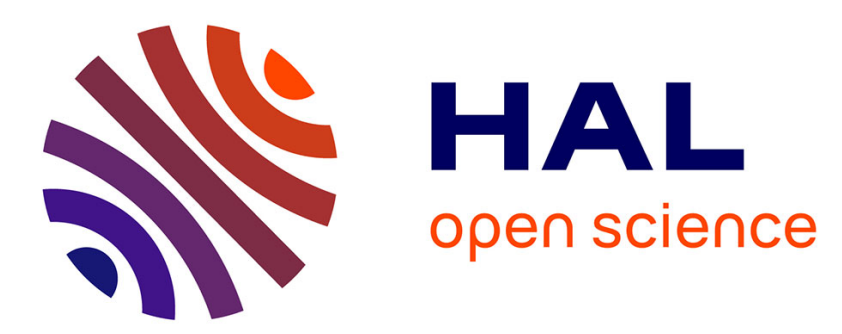

\title{
Traitement Visuel chez l'Enfant Prématuré et Atteinte du Système Magnocellulaire/Dorsal : Synthèse et Perspectives
}

\author{
Mathilde Muneaux, Stéphanie Ducrot
}

\section{To cite this version:}

Mathilde Muneaux, Stéphanie Ducrot. Traitement Visuel chez l'Enfant Prématuré et Atteinte du Système Magnocellulaire/Dorsal: Synthèse et Perspectives. Revue de Neuropsychologie, 2014, 1, pp.17-24. hal-01485960

\author{
HAL Id: hal-01485960 \\ https://hal.science/hal-01485960
}

Submitted on 3 Feb 2022

HAL is a multi-disciplinary open access archive for the deposit and dissemination of scientific research documents, whether they are published or not. The documents may come from teaching and research institutions in France or abroad, or from public or private research centers.
L'archive ouverte pluridisciplinaire HAL, est destinée au dépôt et à la diffusion de documents scientifiques de niveau recherche, publiés ou non, émanant des établissements d'enseignement et de recherche français ou étrangers, des laboratoires publics ou privés. 
Traitement visuel chez l'enfant prématuré et atteinte du système magnocellulaire/dorsal : Synthèse et Perspectives

Abnormal Magnocellular Pathway Visual Processing in preterm children: Assessment and Prospects

\author{
Mathilde Muneaux ${ }^{1,2}$ \\ Stéphanie Ducrot ${ }^{1}$
}

${ }^{1}$ Aix-Marseille Université, CNRS, LPL UMR 7309, 13100, Aix-en-Provence, France.

${ }^{2}$ Centre Hospitalier de Digne-les-Bains, Digne-les-Bains, France

Auteur correspondant :

Mathilde Muneaux

Laboratoire Parole et Langage

CNRS \& Université d'Aix-Marseille

5 avenue Pasteur

13100 Aix-en-Provence

$0413553637 / 0621970733$

$\underline{\text { mathilde.muneaux@1pl-aix.fr }}$ 


\section{RESUME}

Le canal visuel est une entrée essentielle pour l'acquisition de connaissances scolaires. La population grandissante des enfants nés prématurément présente fréquemment ces deux difficultés : problèmes d'acquisitions scolaires (notamment la lecture) et traitement visuel inopérant. Cet article a pour objectif de clarifier au mieux la spécificité du traitement visuel de certains enfants prématurés. Pour ce, nous nous appuyons sur des études comportementales et neurophysiologiques qui excluent les enfants porteurs d'atteintes neurologiques ou périphériques pouvant être à l'origine d'un traitement visuel déficients chez ces enfants. Etant donné que les conditions de maturité cérébrale et d'expérience environnementale précoce sont différentes chez le prématuré, il n'est pas surprenant que le traitement visuel de ces enfants se construise différemment de celui d'enfants nés à terme. Un consensus semble émerger de l'ensemble des données électrophysiologiques et comportementales présentées, à savoir un déficit du traitement des informations visuelles liées au mouvement, à la direction et à la perception d'une forme globale, informations spécifiquement traitées par les voies magnocellulaire/dorsale. Ces informations étant traitées par un système neuronal spécifique, la prématurité pourrait ainsi entraîner des altérations potentielles du développement de ces voies chez les enfants prématurés. Les répercussions possibles sur la scolarité ultérieure de ces enfants seront discutées, en nous appuyant sur un exemple, bien documenté dans la littérature scientifique, du lien entre un dysfonctionnement du système magnocellulaire et une compétence scolaire spécifique : la lecture. 


\begin{abstract}
The visual channel is an essential entry for the acquisition of school knowledge. The growing population of children born prematurely often encounters two difficulties: learning problems (in particular reading disabilities) and ineffective visual processing. The present paper attempts to clarify the specific features of visual processing in preterm children. We refer to behavioral and neurophysiological studies that exclude children with neurological or peripheral disorders likely to be responsible for a visual processing deficit in childhood. Prematurity necessarily entails conditions of neuronal maturity and early-environment experience that differ from those of full-term births. The visual modality is particularly sensitive to these differences, so it is not surprising that premature children's visual processing develops differently from that of full-term children. We discuss the possibility that an abnormal magnocellular pathway visual processing in preterm children could lead to poor academic achievement, taking the example well documented in the scientific literature of selective magnocellular deficits in developmental dyslexia.
\end{abstract}

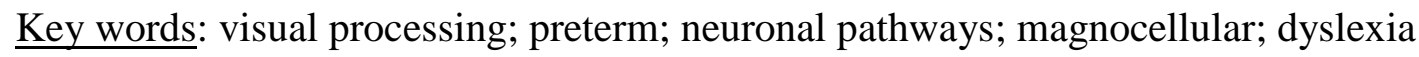


Dans un monde où les écrans sont dorénavant omniprésents, le canal visuel est devenu une entrée primordiale pour accéder aux connaissances. Ce constat est inévitablement le même à l'école. En effet, c'est en regardant des images, des dessins, des vidéos, en lisant... que l'enfant apprend. Traiter toutes ces informations visuelles lui permet de comprendre et donc de stocker en mémoire durablement des connaissances scolaires. La population des enfants nés prématurément a fréquemment ces deux difficultés : problèmes d'acquisitions scolaires [1] et traitement visuel inopérant [2]. Un enfant est considéré prématuré s'il naît avant 37 semaines d'aménorrhée révolues [3]. En 2010, plus d'un enfant sur dix dans le monde est né prématurément, soit 15 millions de nouveau-nés, ce nombre ayant tendance à augmenter dans pratiquement tous les pays [4]. Il se trouve que ces enfants présentent souvent des troubles dans le traitement des informations visuelles. Ces troubles sont fréquemment la conséquence de détériorations organiques d'origine périphérique (e.g., une rétinopathie) ou neurologique (e.g., des lésions cérébrales des voies optiques ou des aires associées) [5]. Mais, certains enfants prématurés ont pourtant un traitement visuel inopérant alors qu'ils ne présentent aucune anomalie d'origine périphérique ou neurologique visible à l'imagerie cérébrale La prématurité étant un élément anamnestique commun à plusieurs pathologies (e.g., dyslexie, dyspraxie, dysphasie, TDAH) [6], mieux comprendre l'origine et les manifestations des difficultés de traitement visuel des enfants prématurés, indemnes de toute anomalie anatomique visible, est un enjeu particulièrement important pour pouvoir aider ces enfants dans leur scolarité. Cependant, il est important, pour la lecture de cet article, de garder à l'esprit le fait qu'une lésion ne soit pas visible à l'imagerie ne signifie pas pour autant une absence de lésion. La limitation actuelle de l'imagerie cérébrale est d'ailleurs fréquemment discutée dans la littérature scientifique sur les troubles neurovisuels [2]. Cet article se propose donc de préciser les difficultés de traitement visuel d'enfants prématurés ne présentant pas d'atteintes neurobiologiques visibles, dans les limites actuelles de l'imagerie. Pour ce faire, un 
rappel succinct de la conception actuelle du système visuel nous permettra d'abord de présenter la spécificité des voies corticales visuelles (i.e., voies magnocellulaire/dorsale et parvocellulaire/ventrale). Ensuite, nous aborderons le développement normal de ces voies ainsi que les conséquences d'une naissance prématurée sur ce développement. L'ensemble des études électrophysiologiques et comportementales présentées suggère que la naissance prématurée entraverait la maturation fonctionnelle du système magnocellulaire/dorsal, ce qui pourrait se répercuter ultérieurement sur la scolarité de ces enfants. Enfin, nous exposerons les travaux déjà réalisés sur le lien entre voies magnocellulaire/dorsale et problèmes d'acquisition scolaires. Nous illustrerons ce lien en prenant l'exemple, bien documenté dans la littérature scientifique, de 1'hypothèse d'un déficit magnocellulaire [7] entravant l'acquisition de la lecture, activité cognitive fréquemment problématique dans la population des prématurés [6].

\section{Conception actuelle du système visuel}

Pour comprendre la spécificité du traitement visuel de certains enfants prématurés, il est primordial au préalable d'aborder la conception actuelle des structures anatomiques soustendant ce traitement $[8,9]$. Le bref descriptif proposé n'a pas la prétention de rendre compte de l'incroyable complexité du système visuel mais permettra de mieux cerner la spécificité du traitement visuel de certains enfants prématurés pourtant indemnes d'anomalies anatomiques.

D’abord, les rayons lumineux émis par l'environnement extérieur sont captés par l'œil. Ils traversent les différents milieux transparents (cornée, humeur aqueuse, cristallin, corps vitré) avant de se projeter sur la rétine, plus précisément sur les cellules photo-réceptrices. Deux types de cellules sont alors mobilisés : les cônes et les bâtonnets. Les cônes (environ 5 millions), principalement concentrés au centre de la rétine (i.e., la fovéa), traitent spécifiquement les informations lumineuses et chromatiques. Les bâtonnets, beaucoup plus nombreux (environ 120 millions), se distribuent sur le reste de la rétine et sont plus sensibles 
aux contrastes. Ces deux types de cellules ont pour objectif commun d'effectuer la transduction du stimulus lumineux en impulsion électrique. Cette information transite alors via les cellules bipolaires et ganglionnaires, puis est acheminée par les axones des cellules ganglionnaires (i.e., les fibres du nerf optique). Les nerfs optiques de chaque œil se rencontrent alors, formant le chiasma optique. Au-delà du chiasma optique, on ne parle plus de nerfs optiques mais de bandelettes optiques qui, contrairement aux nerfs optiques, contiennent des fibres provenant des deux yeux. La grande majorité de ces fibres sont projetées sur les corps genouillés latéraux, situés au niveau du thalamus, constituées majoritairement d'un ensemble de petites cellules, dîtes parvocellulaires, et de grandes cellules, dîtes magnocellulaires, organisées en couches. Le système parvocellulaire traiterait spécifiquement les stimuli chromatiques, les hautes fréquences spatiales et les basses fréquences temporelles. Ce système serait plus lent mais aurait une haute résolution spatiale. Il permettrait, par exemple, de traiter les détails fins d'une image. Le système magnocellulaire traiterait plus particulièrement le mouvement, les faibles fréquences spatiales et les hautes fréquences temporelles. Ce système serait rapide mais aurait une faible résolution spatiale. Il permettrait, entre autres, de percevoir les configurations, les formes et les mouvements rapides. A la sortie des corps genouillés latéraux, les fibres nerveuses constituent les radiations optiques qui se projettent sur le cortex occipital (i.e., aire primaire). Alors, l'information visuelle, apportée par les deux voies parvo- et magnocellulaire, serait intégrée. Cependant, la ségrégation fonctionnelle présente en amont de l'aire primaire, resterait présente en aval donnant naissance aux voies corticales dorsale et ventrale. La voie magnocellulaire fournirait alors l'information dominante à la voie dorsale et la voie parvocellulaire à la voie ventrale. La voie dorsale rejoint les régions postérieures des aires visuelles pariétales, alors que la voie ventrale rejoint les aires visuelles temporales. La voie dorsale serait impliquée pour localiser les objets dans l'espace (traitement visuo-spatial, voie 
du «où »), alors que la voie ventrale serait engagée pour identifier l'objet (traitement identificatoire, voie du « quoi ») [10,11].

Notons que les voies magnocellulaire et dorsale ainsi que les voies parvocellulaire et ventrale traiteraient les mêmes informations visuelles mais à des étapes différentes. Ces voies sont donc extrêmement liées: dépendantes de structures corticales rétro-chiasmatiques et traitant des informations similaires. Il est actuellement difficile de déterminer précisément si un traitement visuel est influencé par les voies présentes en amont (i.e., voies magnocellulaire et parvocellulaire) ou en aval du cortex visuel (i.e., respectivement voies dorsale et ventrale). Par contre, il est à ce jour raisonnable de considérer que les évènements visuels sont traités de deux manières: par les voies magnocellulaire/dorsale sensibles notamment à la forme globale, aux informations visuo-spatiales et aux mouvements ou par les voies parvocellulaire/ventrale particulièrement sensibles aux détails et aux informations chromatiques permettant l'identification de l'objet. Cette dissociation servira de fil conducteur pour traiter du dysfonctionnement du traitement visuel chez certains enfants prématurés qui n'ont aucun anomalie anatomique, anté- ou post-chiasmatique, visible à l'imagerie.

\section{Développement des voies visuelles chez le sujet normal et prématuré}

Pour étudier le développement des voies visuelles chez le nouveau-né, les potentiels évoqués sont particulièrement utiles puisqu'ils ne nécessitent pas de réponses comportementales. En effet, mesurer les changements de l'activité électrique à la surface du crâne permet d'observer dès la naissance les réponses électrophysiologiques à certains types de stimuli visuels. Certains auteurs ont eu recours à cette technique en utilisant des stimuli visuels changeant d'orientation ou de direction pour étudier le développement des voies corticales visuelles (figure 1) [12, 13]. Ils s'appuyaient sur la dissociation conceptuelle 
présentée précédemment : les informations relatives à la direction d'un mouvement seraient traitées par les voies magnocellulaire/dorsale alors que les informations relatives à l'orientation d'une forme seraient traitées par les voies parvocellulaire/ventrale. La présence de potentiels évoqués en réaction à l'orientation étaient observées plus précocement dans le développement normal (dès 3 à 6 semaines après la naissance) mais plus tardivement en réponse à la direction (à partir de 8 à 10 semaines après la naissance) $[12,13]$. Les auteurs ont alors émis l'hypothèse, déjà discutée par Atkinson [14], que le système alloué spécifiquement aux informations en lien avec la direction (i.e., voies magnocellulaire/dorsale) serait plus tardivement fonctionnel, donc plus vulnérable, que le système en charge du traitement d'informations en lien avec l'orientation (i.e., voies parvocellulaire/ventrale) [14]. La naissance prématuré pourrait alors altérer la maturation de la voie magnocellulaire/dorsale.

En effet, la prématurité entraine inévitablement des conditions de maturation cérébrale et d'expérience environnementale précoce différentes de la naissance à terme. Grâce à la protection biologique maternelle, un fœetus a normalement une expérience intra-utérine ajustée à ses besoins : adaptation des nutriments, contrôle de la température et des rythmes chronobiologiques [15]... La dernière partie de la vie fœtale est une période intensive de gyrification des circonvolutions cérébrales, d'accroissement des axones et de synaptogenèse [16]. Ainsi, l'expérience intra-utérine permettrait un niveau de maturation neurobiologique nécessaire et suffisant pour traiter de manière optimale les stimulations sensorielles présentes à la naissance. Cependant, les enfants nés prématurément n’ont pas bénéficié d'autant d'expérience intra-utérine que les enfants nés à terme. Ces nouveau-nés ne pourraient pas alors profiter pleinement d'expériences externes précoces permettant une maturation corticale normale. Ainsi, les premières semaines voire les premiers mois de la vie de ces enfants prématurés sont a fortiori marqués d'expériences visuelles très inhabituelles qui pourraient altérer le développement de leur système visuel. 
Certains auteurs ont testé cette hypothèse avec des bébés prématurés, âgés de 6 à 26 semaines, en mesurant les réponses électrophysiologiques en réponse à des stimuli visuels changeant d'orientation ou de direction $[17,18]$. Les données recueillies étaient similaires chez les bébés prématurés et les bébés du même âge lorsque les stimuli changeaient d'orientation. En revanche, les réponses électrophysiologiques des bébés prématurés aux stimuli changeant de direction apparaissaient plusieurs semaines après qu'elles soient survenues chez les bébés nés à terme. Ces résultats confortent cette hypothèse d'une altération des voies magnocellulaire/dorsale chez les nouveau-nés prématurés. Vient alors inévitablement la question des répercussions de cette perturbation sur le développement de l'enfant. Feng et al. [19] ont utilisé les potentiels évoqués avec des anciens prématurés, âgés de 4 à 6 ans. Les auteurs présentaient aux enfants une configuration de bandes noires et blanches, les zones blanches étant périodiquement transformées en zones noires et vice versa. Puisque que l'intensité lumineuse moyenne de l'écran n'était pas modifiée par ce changement, le résultat du signal était considéré comme étant une réponse électrophysiologique au contraste et non à l'intensité de la lumière, impliquant donc plus fortement un traitement visuo-spatial (i.e., implication des voies magnocellulaire/dorsale) qu'un traitement identificatoire (i.e., implication des voies parvocellulaire/ventrale). Lors de la présentation de ce type de stimuli, les enfants nés prématurément ont montré des réponses électrophysiologiques plus faibles que leurs pairs. Ces résultats vont dans le sens d'une perturbation du développement des voies magnocellulaire/dorsale qui perdurerait bien après la naissance prématurée.

Ainsi, en gardant à l'esprit les limitations actuelles des technologies d'imagerie, l'ensemble de ces études électrophysiologiques suggère que les difficultés de certains enfants nés prématurés à traiter des informations visuelles à un niveau global ou en mouvement seraient liées à une défaillance des voies magnocellulaire/dorsale. Cette particularité serait 
présente dès la naissance et perdurerait tout au long du développement (au moins jusqu'à 9 ans). La naissance prématurée entraverait la maturation fonctionnelle du système magnocellulaire/dorsal, entrainant nécessairement des difficultés pour traiter les informations visuelles présentes dans l'environnement. Nous proposons donc de présenter en détail les études comportementales évaluant le traitement visuel de l'enfant prématuré.

\section{Etudes comportementales du traitement visuel chez l'enfant prématuré}

La majorité des études comportementales s'intéressant au traitement visuel d'enfants prématurés sans atteinte organique visible à l'imagerie s'appuie sur des tests issus de batteries développementales, créées pour l'examen clinique d'enfants présentant des difficultés scolaires, et non pour l'évaluation spécifique du traitement visuel. Il en résulte une grande hétérogénéité des résultats [20-23]. En effet, l'utilisation de ces épreuves implique de nombreux processus, autres que le traitement visuel, influençant aussi les performances. Par exemple, le test Triangles du KABC II [24], nécessitant d'effectuer une construction de triangles en référence à un modèle en deux dimensions, requière, en plus de compétences motrices, de nombreuses capacités cognitives telles que la planification, les compétences praxiques, le traitement visuo-spatial... qui influencent inévitablement les performances. Nous avons donc choisi de ne pas présenter ces études mais plutôt de s'attarder sur des études, certes plus rares, mais utilisant une méthodologie expérimentale ciblant spécifiquement la particularité du traitement visuel chez l'enfant prématuré indemne d'atteintes anatomiques visibles à l'imagerie.

L'une d'entre elles a utilisé des tâches mettant en œuvre deux types de traitement de l'information visuelle avec des enfants de 6 à 9 ans nés prématurément [25]. Un traitement impliquait les parties d'un stimulus visuel sans prendre en considération le stimulus dans son ensemble (i.e., traitement local). L'autre engageait le traitement de la forme du stimulus sans 
prendre en considération les éléments distincts qui la composent (i.e., traitement global). Une tâche visuo-contructive consistait à demander aux enfants de recopier des stimuli « lettre »ou «forme», constitué de petits lettres ou formes (niveau local) formant une autre lettre ou forme (niveau global). La qualité de la production était évaluée aux niveaux local et global. Une autre tâche, dite visuo-perceptive, consistait à demander aux enfants de choisir le plus rapidement possible parmi deux figures laquelle ressemblait le plus à une figure modèle. Chaque figure était composée d'éléments (lettres ou formes) organisés en un tout (lettre ou forme). Les figures proposées ressemblaient au modèle (e.g., un carré composé de petits carrés) d'un point de vue global (e.g., un carré composé de petits cercles) ou local (e.g., un cercle composé de petits carrés). Pour la tâche visuo-perceptive, les auteurs ont observé le même comportement chez les enfants prématurés que chez leurs pairs, à savoir une tendance à se baser préférentiellement sur le niveau global pour établir une comparaison. Par contre, pour la tâche visuo-constructive, les enfants nés prématurément dessinaient aussi bien les éléments locaux que leurs pairs, mais ils étaient beaucoup moins précis pour composer la forme globale (figure 2). Dans cette étude, la composante motrice ne pouvait rendre compte de cette différence puisque les deux groupes étaient équivalents pour dessiner les éléments locaux. Cette étude laisse supposer que certains enfants nés prématurés auraient des difficultés pour traiter les informations dans leur ensemble (i.e., traitement allouée aux voies magnocellulaire/dorsale).

L'étude de Taylor et al. [26] a permis d'apporter des éléments supplémentaires en utilisant une tâche similaire de détection de forme globale (figure 3) mais aussi une tâche dynamique de détection globale de mouvements. Cette dernière consistait à repérer si des points en mouvement sur un écran d'ordinateur montaient ou descendaient. Les enfants prématurés de 5 à 9 ans avaient besoin de plus de points pour détecter un mouvement ou une forme que leurs pairs. Ce résultat est conforté par l'étude de MacKay et al. [27] ayant recours 
à trois tâches de détection de mouvements chez des enfants nés prématurés de 5 à 8 ans : une tâche de détection globale de mouvements similaire à celle de Taylor et al. [26] et deux tâches de détection locale de mouvements qui requéraient aussi la détection d'un mouvement ascendant ou descendant. Dans l'une des tâches locales, le mouvement était induit par le changement de luminosité dans le temps (figure 4), alors que dans l'autre tâche, le mouvement était produit par des variations spatio-temporelles de l'image comme le contraste (e.g., une région de haut contraste se déplaçant à travers une région de plus faible contraste mais de même luminosité, figure 4). Les enfants prématurés présentaient plus de difficultés pour détecter les mouvements que des enfants du même âge pour les trois tâches de détection de mouvements (i.e., informations traitées par les voies magnocellulaire/dorsale).

L'ensemble des données comportementales semble donc aller dans le sens d'un dysfonctionnement de ce système magnocellulaire/dorsal. Il est alors possible d'émettre l'hypothèse qu'un tel dysfonctionnement pourrait se répercuter sur la scolarité des enfants nés prématurés. En effet, comme le souligne Gaillard [28] «voir n'est jamais une aptitude isolée ». La perception visuelle, en lien avec les autres modalités sensorielles, contribue à donner sens, à conceptualiser ce qui est perçu et à s'adapter de manière optimale à l'environnement. Par conséquent, le développement de l'enfant, qu'il soit cognitif ou moteur, est nécessairement lié au développement de ses capacités à traiter les informations sensorielles. Il est donc raisonnable d'envisager qu'un traitement visuel inadapté pourrait avoir une part de responsabilité dans les difficultés que présentent nombre d'anciens prématurés dans leur milieu scolaire et professionnel [1, 6, 29]. Par exemple, la méta-analyse de Bhutta et al. [30] ainsi que l'étude EPIPAGE ${ }^{1}$ [21] font état d'une perte d'environ 10 points de QI (Quotient Intellectuel, indice utilisé dans les échelles d'efficience intellectuelle

\footnotetext{
${ }^{1}$ Etude EPIdémiologique sur les Petits Ages Gestationnels menée de 1997 à 2002 sur 1808 enfants prématurés nés avant 32 semaines d'aménorrhée. En 2011, une étude EPIPAGE 2 a été lancée et sera poursuit sur une période de 11-12 ans.
} 
de Wechsler) pour des enfants nés prématurément par rapport à des enfants nés à terme. En outre, de nombreux enfants prématurés présentent des difficultés allant des troubles de coordination visuo-motrices [23] aux troubles du développement du langage écrit ou oral [6, 31]. Le chapitre suivant se propose d'illustrer ce lien entre déficit des voies magnocellulaire/dorsale et problèmes d'acquisition scolaire en présentant les répercussions possibles d'une atteinte de la voie magnocellulaire sur une activité cognitive particulièrement importante pour les acquisitions scolaires (en l'occurrence la lecture) [7].

\section{Lecture et système magnocellulaire chez le sujet normal et dyslexique}

Une caractéristique importante du système visuel magnocellulaire est sa capacité à traiter, outre les faibles fréquences spatiales, le mouvement et les changements rapides ; les cellules magnocellulaires communiquent très rapidement toute information de mouvement au cortex visuel via les couches magnocellulaires du corps genouillé latéral, du thalamus et au colliculus supérieur pour le contrôle réflexe des mouvements oculaires, permettant ainsi de guider les yeux vers un point précis, tout en filtrant les informations non pertinentes. La mobilité du regard est nécessairement fortement engagée dans l'activité de lecture mais elle n'est toutefois pas le seul moteur de cette vision fine impliquée dans le traitement de l'écrit. Les mouvements des yeux et l'attention visuelle sont aussi impliqués. Ces capacités visuoattentionnelles fines seraient particulièrement importantes pour traiter l'écrit. En effet, l'œil ne peut voir l'ensemble d'un texte, il ne peut voir que ce qui se trouve autour de ce qu'il fixe. Cette zone de perception est appelée fenêtre attentionnelle. Certains auteurs considèrent que cette fenêtre serait ajustée en fonction du type de mot lu, le traitement de mots familiers mobilisant des fenêtres de plus grande taille que le traitement de mots nouveaux [32, 33]. Chez le lecteur expert, le traitement des mots se ferait d'abord en mode global avec une fenêtre visuo-attentionnelle qui englobe l'ensemble du mot traité [34]. Cependant, ce mode de 
traitement échouerait lorsqu'aucune représentation du mot lu n'est stockée en mémoire. Alors, lorsque le traitement porte sur un mot non connu, le système basculerait en mode analytique. Dans ce mode, la fenêtre visuo-attentionnelle se réduirait pour traiter les unités sublexicales (graphèmes ou syllabes) qui composent le mot. Comme la fenêtre est restreinte, de nombreux déplacements de l'œil seraient alors nécessaires pour traiter le mot en son entier. D'autres auteurs postulent que cette fenêtre attentionnelle serait fonction d'un certain nombre de contraintes anatomo-fonctionnelles du système visuel [35]. En s'appuyant sur l'étude des mouvements oculaires, ces auteurs postulent que la fenêtre visuo-attentionnelle des lecteurs experts serait limitée à la ligne de texte en train d'être lue et s'étendrait approximativement du début du mot fixé à 14-15 caractères à droite, cette asymétrie à droite n'étant valable que pour des langues se lisant de gauche à droite [36]. A l'intérieur de cette fenêtre, deux types de traitement seraient effectués. Un traitement très précis et de haute acuité autour de la position fixée par l'œil permettrait l'identification du mot (traitement fovéal). Un traitement plus large et plus loin de la position fixée renseignerait sur la forme et la longueur des mots suivants pour guider l'œil et déterminer le prochain lieu de fixation (traitement parafoveal). De nombreuses recherches ont montré que lorsque des lecteurs experts lisent un mot, ils positionnent systématiquement leur œil sur une zone à mi-chemin entre le début et le milieu du mot $[37,38]$. Cette position permettrait d'optimiser le traitement et par conséquent l'accès à la représentation du mot écrit stockée en mémoire (i.e., position optimale du regard ou Optimal Viewing Position) [37]. Lors de l'apprentissage de la lecture, l'enfant va devoir apprendre à positionner son œil correctement sur les mots, à extraire l'information qui est en train d'être fixée, à programmer une saccade pour se positionner sur le mot suivant [39]... Un dysfonctionnement de ces capacités visuo-attentionnelles pourrait donc entrainer des difficultés dans l'acquisition du langage écrit. 
Cette idée selon laquelle un déficit visuel indépendamment de toute atteinte sensorielle (ou périphérique) pourrait entraver l'apprentissage de la lecture est d'ailleurs très ancienne puisque le tout premier cas d'enfant dyslexique décrit dans la littérature scientifique parlait de «cécité verbale congénitale »[40]. En 1991, des données histologiques recueillies postmortem sur le cerveau de cinq personnes préalablement identifiées dyslexiques ont par la suite conforté cette hypothèse [41]. Cette étude montrait que les cellules du système magnocellulaire au niveau du corps genouillé latéral avaient des corps cellulaires de taille plus réduite chez les dyslexiques ( $27 \%$ plus petits) que chez leurs pairs. Rappelons que la dyslexie est une pathologie développementale entravant l'acquisition de la lecture en l'absence de déficience intellectuelle, de troubles sensoriels ou psychiatriques et malgré un enseignement conventionnel. De nombreuses études ont montré des perturbations de la vision des contrastes et de la sensibilité au mouvement chez les dyslexiques, évoquant alors l'hypothèse d'un déficit du système magnocellulaire [7, 42, 43]. De plus, plusieurs recherches ont rapporté chez les dyslexiques la présence d'une instabilité de la fixation oculaire engendrant des distorsions, des déplacements et des superpositions de lettres et de mots [44, 45], ainsi que des troubles de la poursuite oculaire, essentiellement de gauche à droite [46]. En outre, un déficit magnocellulaire chez les dyslexiques n'a pas été observé seulement pour la motilité du regard ou lors de traitement des basses fréquences. En effet, des études comportementales mettent en évidence un dysfonctionnement des capacités visuo-attentionnelles chez les dyslexiques. Par exemple, Bosse et ses collaborateurs [32] ont montré qu'un groupe d'enfants dyslexiques percevaient moins de lettres qu'un groupe contrôle lorsqu'il traitait une suite de consonnes (e.g., RHSDM). D'autres études ont montré que lors de l'activité de lecture, les enfants dyslexiques ne positionnaient pas toujours leur regard sur le point idéal (i.e., optimal viewing position), entraînant des erreurs de positionnement trop fréquentes et occasionnant alors une augmentation des re-fixations [47]. Ainsi, non seulement les enfants dyslexiques ne fixeraient 
pas le mot de manière optimale mais en plus ils ne traiteraient pas efficacement l'information visuelle située en périphérie [48]. Ce phénomène « d'encombrement perceptif » (i.e., crowding effect) est d'ailleurs très présent dans la population des personnes dyslexiques [49].

Actuellement, il existe déjà des batteries diagnostiques créées pour détecter, spécifiquement et précocement, les troubles dans le traitement d'informations en lien avec le système magnocellulaire/dorsal (batterie sensible dès l'âge de 4 ans) [50]. De plus, des rééducations ciblées sur les capacités visuo-attentionnelles se sont avérées efficaces chez certains enfants dyslexiques [51, 52]. Plus récemment, il a été montré que l'augmentation de l'espacement des lettres d'un mot et des mots d'un texte, réduisant l'encombrement perceptif, améliore la vitesse et la qualité de la lecture chez certains enfants dyslexiques. Ainsi, en augmentant cet espacement, des enfants dyslexiques, n'ayant pourtant bénéficié d'aucun entraînement préalable, lisaient en moyenne $20 \%$ plus vite et faisaient deux fois moins d'erreurs que lorsqu'ils lisaient un texte avec un espacement standard [49].

\section{Conclusion et perspectives}

Un consensus semble émerger de l'ensemble des études présentées dans cet article : les personnes nés prématurés présentent des difficultés de traitement des informations liées au mouvement, à la direction et à la perception d'une forme globale, informations spécifiquement traitées par les voies magnocellulaire/dorsale. Les conséquences d'un traitement visuel inopérant chez les prématurés pourraient dès lors être traitées à l'aide des batteries et des aménagements spécifiques existant déjà pour dépister et aider les enfants dyslexiques présentant des difficultés en lien avec un déficit des voies magnocellulaire et/ou dorsal. Cependant, d'autres troubles fréquemment présents dans la population des enfants prématurés (i.e., moteurs ou langagiers) ne bénéficient pas encore de telles techniques. Ainsi, continuer à préciser les caractéristiques neuro-développementales des enfants prématurés 
permettrait de développer des outils nouveaux de dépistage et de prise en charge afin d'aider l'ensemble des prématurés en situation d'échec scolaire [53]. Cependant, au préalable, il est nécessaire de développer les techniques actuelles d'imagerie cérébrale pour préciser les spécificités du fonctionnement neurologique des enfants prématurés et mieux les spécificités de l'anatomie cérébrale de ces enfants. En effet, rappelons que l'absence de lésions visibles à l'imagerie ne signifie pas forcément que les structures neuro-anatomiques sont indemnes d'anomalies mais pourrait juste être le reflet des limites de la technologique actuelle. Il convient donc de rester prudent quant aux interprétations émises à partir des données électrophysiologiques présentées précédemment. A noter toutefois que des avancées technologiques récentes présentent des perspectives intéressantes pour dépasser ces limitations. Par exemple, la spectroscopie proche infra-rouge permet de mesurer en temps réel les concentrations locales d'hémoglobine oxygénée et désoxygénée dans les tissus en déterminant le degré d'absorption de lumière proche de l'infrarouge. La magnétoencéphalographie (MEG) quant à elle, mesurant des champs magnétiques induits par l'activité électrique des neurones du cerveau en réponse à une stimulation externe, a déjà été réalisée sur le foetus in utéro [54]. Même si ces techniques n’ont pas encore été employées pour étudier le traitement visuel dans le cadre de la prématurité [55], il est possible d'envisager que leur utilisation permettrait de préciser les aspects morphologiques et fonctionnels du cerveau des personnes nés prématurés et ainsi d'apporter des éléments supplémentaires au débat actuel. 


\section{REFERENCES}

1. Sayeur MS, McKerral M, Lassonde M. Les troubles cognitifs, comportementaux et d'apprentissage chez les enfants nés prématurément : une revue de littérature. Neuropsicol Lat am $2011 ; 3(2)$ : 14-22.

2. Ortibus EL, De Cock PP, Lagae LG. Visual Perception in Preterm Children : What Are We Currently Measuring ? Pediatr Neurol 2011 ; 45(1) : 1-10.

3. WHO : recommended definitions, terminology and format for statistical tables related to the perinatal period and use of a new certificate for cause of perinatal deaths. Modifications recommended by FIGO as amended October 14, 1976. Acta Obstet Gynecol Scand 1977 ; 56(3) : 247-53.

4. OMS. Born to soon, the global action report on Preterm Birth. Téléchargeable sur http://whqlibdoc.who.int/publications/2012/9789241503433_eng.pdf, 2012.

5. Guzzetta A, Cioni G, Cowan F, et al. Visual disorders in children with brain lesions: 1 . Maturation of visual function in infants with neonatal brain lesions: correlation with neuroimaging. Eur J Paediatr Neurol 2001 ; 5(3) : 107-14.

6. Nosarti C, Murray RM, Hack M. Neurodevelopmental Outcomes of Preterm Birth: From Childhood to Adult. Cambridge (UK) : Cambridge University Press, 2010.

7. Stein J, Walsh V. To see but not to read : the manocellular theory of dyslexia. Trends Neurosci $1997 ; 20: 147-52$.

8. Calabrèse, A. (2011). Désorganisation fonctionnelle des systèmes oculomoteur et visuoattentionnel chez les patients basse vision-Approche psychophysique. Thèse de doctorat en neurosciences cognitives, non publiée, Université de la méditerranée, Marseille.

9. Chokron S, Marendez C. Comment voyons-nous ? Paris : Le Pommier, 2010. 
10. Milner DA, Goodale MA. The visual brain in action. Cambridge (MA) : MIT Press, 1995.

11. Ungerleider LG, Mishkin M. Two cortical visual systems. In : Ingle DJ, Goodale MA, Mansfield RJW, eds. Analysis of visual behavior. Cambridge (MA) : MT Press, 1982 : $549-86$.

12. Braddick OJ. Orientation- and motion-selective mechanisms in infants. In : Simons K, ed. Early visual development: normal and abnormal. New York : Oxford University Press; 1993.

13. Braddick OJ, Birtles D, Wattam-Bell J, et al. Motion and orientation specific cortical responses in infancy. Vis Res 2005 ; 45 : 3169-79.

14. Atkinson J. Early development : differential functioning of parvocellular and magnocellular pathways. Eye $2002 ; 6$ : 129-35.

15. Rivkees SA, Reppert SM. Perinatal development of day-night rhythms in humans, Horm Res $1992 ; 37$ : 99-104.

16. Adams-Chapman I. Neurodevelopmental outcome of the late preterm infant. Clin Perinatol $2006 ; 33: 947-64$

17. Atkinson J, Birtles D, Anker S, et al. High-density VEP measures of global form and motion processing in infants born very preterm. $J$ Vis $2008 ; 8: 422$.

18. Birtles D, Braddick O, Wattam-Bell J, et al. Orientation and motion-specific visual cortex responses in infants born preterm. NeuroReport 2007 ; 18 : 1975-9.

19. Feng J-J, Xu X, Wang W-P, et al. Pattern visual evoked potential performance in preterm preschooler with average intelligence quotients. Early Hum Dev 2011 ; 87 : 61-6. 
20. Geldof CJ, van Wassenaer AG, de Kieviet JF, et al. Visual perception and visual-motor integration in very preterm and/or very low birth weight children : a meta-analysis. Res Dev Disabil 2012 ; 33(2) : 726-36.

21. Larroque B, Ancel P-Y, Marret S, et al. Neurodevelopmental disabilities and special care of 5-year-old children born before 33 weeks of gestation (the EPIPAGE study) : a longitudinal cohort study. Lancet 2008 ; $371: 813-20$.

22. O’Reilly M, Vollmer B, Vargha-Khadem F, et al. Ophthalmological, cognitive, electrophysiological and MRI assessment of visual processing in preterm children without major neuromotor impairment. Dev Sci 2010 ; 13(5) : 692-705.

23. De Kieviet JF, Piek JP, Aarnoudse-Moens CS, et al. Motor development in very preterm and very low-birth-weight children from birth to adolescence : A meta-analysis. JAMA $2009 ; 302: 2235-42$.

24. Kaufman AS, Kaufman NL. Kaufman assessment battery for children, interpretive manual. Circle Pines (MN) : American Guidance Service, 1983.

25. Santos A, Duret M, Mancini J, et al. Preterm birth affects dorsal-stream functioning even after age 6. Brain Cogn 2009 ; 69(3) : 490-4.

26. Taylor NM, Jakobson LS, Maurer D, et al. Differential vulnerability of global motion, global form, and biological motion processing in full-term and preterm children. Neuropsychologia $2009 ; 47$ : 2766-78.

27. MacKay TL, Jakobson LS, Ellemberg D, et al. Deficits in the processing of local and global motion in very low birthweight children. Neuropsychologia $2005 ; 43$ : 1738-48.

28. Gaillard F. Développement de la perception visuelle. Actes des journées de l'A.L.F.P.H.V. Lausanne, 1984. 
29. Marret S, Ancel P-Y, Kaminski M. Prématurité modérée et tardive : devenir neurodéveloppemental des enfants. Archiv Pédiatr $2011 ; 18$ : 41-2.

30. Bhutta AT, Cleves MA, Casey PH, et al. Cognitive and behavioral outcomes of schoolaged children who were born preterm : a meta-analysis, JAMA 2002 ; 288 : 728-37.

31. Charollais A, Stumpf M-H, Beaugrand D, et al. Evaluation à 6 ans du langage de l'enfant né grand prématuré sans paralysie cérébrale : étude prospective de 55 enfants. Archiv Pédiatr $2010 ; 17: 1433-9$.

32. Bosse ML, Tainturier MJ, Valdois S. Developmental dyslexia : the visual attention span deficit hypothesis. Cognition 2007 ; 104 : 198-230.

33. Valdois S, Lassus-Sangosse D, Lobier, M. The visual nature of the visual attention span disorder in developmental dyslexia. In Stein J, Kapula Z, eds. Visual aspects of dyslexia. Oxford University Press, $2012 ; 111-22$.

34. Ans B, Carbonnel S, Valdois S. A connectionist multiple-trace memory model for polysyllabic word reading. Psychol Rev $1998 ; 105$ : 678-723.

35. Ducrot S, Lété B. Attention et contrôle oculaire en lecture experte. In Michael G, editor. Neurosciences cognitives de l'attention visuelle. Marseille : Solal, 2008 ; 229-64.

36. Rayner K, Well AD, Pollatsek A. Asymmetry of the effective visual field in reading. Percept Psychophys $1980 ; 27$ : 537-44.

37. O'Regan JK, Levy-Schoen A, Pynte J, et al. Convenient fixation location within isolated words of different length and structure. J Exp Psychol Hum Percept Perform 1994 : 10 ; 250-7.

38. Rayner K. Eye guidance in reading : Fixation location within words. Perception $1979 ; 8$, $1979: 21-30$. 
39. Ducrot S, Pynte J, Ghio A, et al. Visual and Linguistic Determinants of the Eyes' Initial Fixation Position in Reading Development. Acta Psychol 2013 ; 142 (3) : 287-298.

40. Morgan WP. A case of congenital word blindness. British Medical Journal 1896 : 2 ;1378.

41. Livingstone MS, Rosen GD, Drislane FW, et al. Physiological and anatomical evidence for a magnocellular defect in developmental dyslexia. Proceedings of the National Academy of Science $1991 ; 88$ :7943-7.

42. Cornelissen PL, Richardson AJ, Mason AJ, et al. Contrast sensitivity and coherent motion detection measured at photopic luminance levels in dyslexics and controls. Vis Res 1995 ; 35(10) : 1483-94.

43. Lovegrove, WJ, Martin F, Slaghuis W. A theoretical and experimental case for a visual deficit in specific reading disability. Cogn Neuropsychol $1986 ; 3$ : 225-267.

44. Chase C, Stein J. Visual magnocellular deficits in dyslexia. Brain 2003 ;126(Pt 9): E2; author reply E3.

45. Stein J. The magnocellular theory of developmental dyslexia. Dyslexia $2001 ; 7(1)$ : 1236.

46. Eden GF, Stein JF, Wood HM, Wood FB. Differences in eye movements and reading problems in dyslexic and normal children. Vision Res 1994 ; 34(10) :1345-58.

47. Hawelka S, Gagl B, Wimmer H. A dual-route perspective on eye movements of dyslexic readers. Cognition $2010 ; 115: 367-79$.

48. Ducrot S, Lété B, Sprenger-Charolles L, et al. The viewing position effect in beginning and dyslexic readers. Current Psychology Letters, Behaviour, Brain and Cognition 2003 $; 10(1): 1-13$. 
49. Zorzi M, Barbiero C, Facoetti A, et al. Extra-large letter spacing improves reading in dyslexia, Proc Natl Acad Sci 2012 ; 109(28) : 11455-9.

50. Cavézian C, Vilayphonh M, de Agostini M, et al. Assessment of visuo-attentional abilities in young children with or without visual disorder: toward a systematic screening in the general population. Res Dev Disabil 2010 ; 31(5) : 1102-8.

51. Launay L, Valdois S. Evaluation et prise en charge cognitive de l'enfant dyslexique et/ou dysorthographique de surface. In : Valdois S, Colé P, David D, eds. Apprentissage de la lecture et dyslexies développementales : de la théorie à la pratique. Marseille : Solal, $2004: 209-32$.

52. Lobier M, Valdois S. Prise en charge des dyslexies développementales : critères d'évaluation, Rev Neuropsychol $2010 ; 1$ : 102-9.

53. Chyi LJ, Lee HC, Hintz SR, et al. School outcomes of late preterm infants : special needs and challenges for infants born at 32 to 36 weeks gestation. J Pediatr $2008 ; 153$ : 25 31.

54. Sheridan CJ, Matuz T, Draganova R, et al. Fetal Magnetoencephalography Achievements and Challenges in the Study of Prenatal and Early Postnatal Brain Responses : A Review. Infant Child Dev 2010 ; 19(1) : 80-93.

55. Mento G, Bisiacchi PS. Neurocognitive development in preterm infants : insights from different approaches. Neurosci Biobehav Rev 2012 ; 36 : 536-55. 